\title{
A Measure of Peripheral Nerve Stimulation Efficacy Applicable to H-Reflex Studies
}

\author{
G.I. Boorman, J.A. Hoffer, K. Kallesoe, D. Viberg and C. Mah
}

\begin{abstract}
Background: When H-reflexes are recorded during movement in human subjects, the stimulator current output is not a good indicator of sensory stimulation efficacy because of unavoidable nerve movement relative to the stimulus electrodes. Therefore, the $\mathrm{M}$-wave amplitude has been used by researchers as an indicator of the efficacy of the stimulus. In this study we have examined the general validity of the hypothesis that the $\mathrm{M}$-wave amplitude is directly proportional to the group I sensory afferent volley evoked by the stimulus. Methods: A nerve recording cuff, stimulating electrodes, and EMG recording electrodes were implanted in cats. Nerve cuff recordings of centrally propagating volleys evoked by electrical stimuli were directly compared to $\mathrm{M}$-waves produced by the same stimuli. Compound action potentials (CAPs) recorded in the sciatic nerve were compared with soleus M-waves during either tibial nerve or soleus muscle nerve stimulation. CAPs in the ulnar nerve were correlated with flexor carpi ulnaris M-waves during ulnar nerve stimulation. Results and Conclusions: Our findings indicate that for mixed nerve stimulation (e.g., tibial or ulnar nerve) the M-wave can be a reliable indicator of the centrally propagating sensory volley. Due to the high correlation between CAP and Mwave amplitude in these nerves, a small number of $M$-waves can give a good estimate of the size of the group I sensory volley. On the other hand, when nerves with only partially overlaping fibre diameter populations are stimulated (e.g., the soleus muscle nerve), the M-wave is not well correlated with the group I sensory volley and thus may not be used as a measure of the size of the input volley for H-reflex studies.
\end{abstract}

RÉSUMÉ: Mesure de l'efficacité de la stimulation d'un nerf périphérique applicable à l'étude du réflexe $\mathbf{H}$. Introduction: Pendant l'enregistrement du réflexe $\mathrm{H}$ chez l'humain en mouvement, les données de l'électrode stimulatrice ne fournissent pas des renseignements précis sur l'efficacité du stimulus, à cause des mouvements inévitables du nerf par rapport à l'électrode. C'est pourquoi des chercheurs ont utilisé l'amplitude de l'onde $\mathrm{M}$ comme indicateur de l'efficacité du stimulus. Nous avons examiné l'hypothèse suivante: l'amplitude de l'onde $M$ est directement proportionnelle à la volée afférente sensitive du groupe I évoquée par le stimulus. Méthodes: Une manchette d'enregistrement, des électrodes de stimulation ainsi que des électrodes d'enregistrement EMG ont été implantées chez des chats. Les enregistrements générés par le stimulus électrique ont été comparés directement à l'amplitude de l'onde M produite par le même stimulus. Les potentiels d'action composés (CAPs) enregistrés dans le nerf sciatique ont été comparés aux ondes $\mathrm{M}$ du muscle soléaire pendant la stimulation du nerf tibial ou du nerf du muscle soléaire. Les CAPs dans la région proximale du nerf cubital ont aussi été comparés à l'amplitude des ondes $\mathrm{M}$ du muscle cubital antérieur pendant la stimulation du nerf cubital. Résultats et Conclusions: Nos données montrent que pendant la stimulation d'un nerf mixte (sciatique poplité interne ou cubital) l'amplitude de l'onde $M$ peut être un indicateur fiable de la volée sensitive à propagation centrale. La forte corrélation entre le CAP et l'amplitude de l'onde M observée dans l'étude de ces nerfs montre qu'un petit nombre d'ondes $\mathbf{M}$ est suffisant pour donner une bonne appréciation de l'ampleur de la volée sensitive du groupe I. Cependant, dans le cas où le nerf stimulé a des populations de fibres dont les diamètres se chevauchent partiellement comme le nerf du muscle soléaire, l'amplitude de l'onde $\mathrm{M}$ n'est pas bien corrélée à la volée sensitive du groupe I. Ainsi, l'amplitude de l'onde $\mathrm{M}$ ne peut pas être utilisée pour mesurer l'ampleur du stimulus dans l'étude du réflexe $\mathrm{H}$.

Can. J. Neurol. Sci. 1996; 23: 264-270

In order to better understand spinal cord function, researchers have examined reflexes, including the H-reflex, during natural movement. ${ }^{1,2}$ Although $\mathrm{H}$-reflex studies are potentially a valuable tool to investigate spinal mechanisms, there are problems of interpretation. To interpret reflex outputs, it is necessary to control the inputs to spinal circuitry. When H-reflexes are elicited in human subjects performing voluntary movement, the nerve moves within the stimulus field. The resulting changes in the
From the Department of Clinical Neurosciences, The University of Calgary, Calgary, Alberta (G.I.B., C.M.); School of Kinesiology, Simon Fraser University, Burnaby, British Columbia (J.A.H., K.K., D.V.) RECEIVED MAY 23, 1995. ACCEPTED IN FINAL, FORM MAY 8, 1996.

Reprint requests to: Dr. G.I. Boorman, University of California Los Angeles, Dept. Physiological Science, 405 Hilgard, 2322 Life Science Building, Los Angeles. California, USA $90095-1527$ 
stimulus field preclude meaningful estimates of the extent of nerve fibre recruitment from records of stimulator output. In order to validate the results of such studies, it is necessary to demonstrate that changes in reflex amplitude are caused by physiological mechanisms, rather than simply reflecting changes in the stimulus field/nerve geometry.

Several studies that examined H-reflexes during movement used the direct motor response recorded from a muscle (the $\mathrm{M}$ wave) as a simple accessible measure of stimulus strength. ${ }^{2-6}$ Their assumption was that during electrical stimulation of a mixed nerve, the number of afferent fibres with centrally propagating action potentials would be directly proportional to the number of motor fibres with distally propagating action potentials. Thus, stimuli that elicited $\mathrm{M}$-waves of a given amplitude were assumed to also elicit afferent volleys of matched amplitude.

Although generally accepted by investigators, the validity of the $M$-wave as a measure of peripheral nerve stimulus efficacy has remained largely unproven. Poor correlations between the size of the M-wave and the size of the sensory afferent volley could occur for at least three reasons. First, the smoothness of the recruitment curves for sensory and motor fibres will depend on both the spectra of fibre diameters and the relative intrafascicular locations. Second, the recruitment curves may change unpredictably if the stimulation electrodes move with respect to the nerve. Third, even at matched motor axon recruitment levels, the $\mathrm{M}$-wave shape and amplitude may vary markedly as muscle fibre lengths and orientations change during movement.

At present, there is no available way to examine these questions in human experiments. However, direct recordings in moving animals are possible using implanted nerve cuffs. ${ }^{7}$ In this study we examined the validity of using the M-wave as a measure of peripheral nerve stimulus efficacy, by recording simultaneously the nerve compound action potentials (CAPs) and the $\mathrm{M}$-waves in response to peripheral nerve stimulation using implanted electrodes in cats.

Our findings indicate that the validity of using the $\mathrm{M}$-wave as a direct indicator of the size of the centrally propagating group I afferent volley depends on the specific nerve, muscle and reflex pathway studied. For some systems and if appropriate precautions are taken the use of the M-wave is valid, but for other systems this approach may not be appropriate.

\section{METHODS}

Six large male adult cats, selected for their ease of handling and trainability, were individually housed in large cages and cared for according to guidelines of the Canadian Council for Animal Care. Research protocols were approved by The University of Calgary Animal Care Committee prior to commencement of this work. Cats were deeply anaesthetized with Halothane mixed in oxygen. Aseptic surgical techniques were used as described by Hoffer. ${ }^{7}$ During post-surgical treatment, analgesics (Atravet followed by morphine) were administered to minimize any post-operative discomfort. The cats walked without impairment by about four days post surgery.

All electrodes were made from multistranded stainless steel wire coated with Teflon (Cooner AS-631). The following electrodes were implanted in the left hindlimb in five of the six cats (see Figure 1). Bipolar EMG electrodes were implanted in the soleus muscle and a stimulating patch electrode was implanted over the entry point of the soleus nerve. ${ }^{7}$ The soleus nerve stimulating electrodes consisted of a superficially placed wire embedded in a silicone patch and another wire implanted in the soleus muscle deep with respect to the nerve entry point. A bipolar nerve stimulating cuff made from silicone tubing was placed around the tibial nerve proximal to the popliteal fossa. A tripolar nerve recording cuff was placed around the sciatic nerve distal to the branching of the hamstring nerves. A thermistor was implanted in the popliteal fossa and used to monitor limb temperature.?

In the sixth cat, two cuffs on the ulnar nerve (used for stimulation or recording), EMG recording electrodes in flexor carpi ulnaris (FCU) and a thermistor were implanted in the left forelimb. In all the cats, electrode leads coursed subcutaneously to the dorsal lumbar region, emerged percutaneously and were soldered to a 40 pin external connector that was attached with sutures.?

\section{Electrical stimulation}

The interplay between stimulus pulse shape (i.e., width, rise and fall times) and pulse amplitude for nerve fibre recruitment during nerve stimulation is complex..$^{8,9}$ Most human H-reflex studies have used simple monophasic rectangular pulses. In the

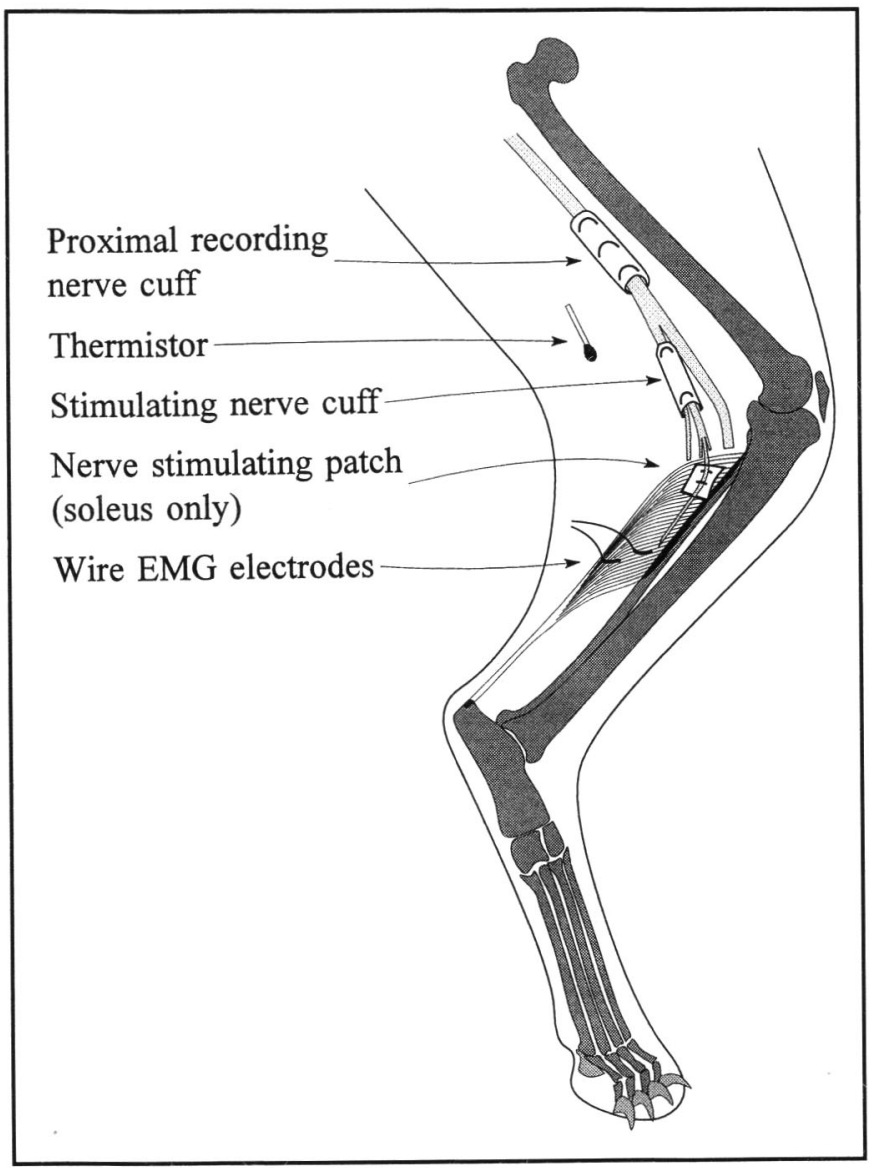

Figure 1: Medial aspect of the left hindlimb. CAPs were recorded with a tripolar recording cuff implanted around the whole sciatic nerve. Soleus EMG ( $M$-wave) was recorded with indwelling wire leads. A bipolar stimulating cuff was implanted on the nerve between the two recording sites. In some hindlimb experiments, additionally a stimulating patch was sewn on the muscle at the nerve entry point to selectively stimulate the soleus muscle nerve. 
present study, monophasic rectangular pulses $0.1 \mathrm{~ms}$ in duration, negative polarity were used, and each stimulus pulse was immediately followed with a second pulse of opposite polarity, $1 \mathrm{~ms}$ in duration and $1 / 10$ the amplitude of the stimulus pulse. This approach had the desirable quality of balancing total "charge injection", ${ }^{10}$ thus reducing the risks of tissue toxicity as well as etching of the electrodes.

\section{Recording protocols}

During recording sessions the cats were quietly sitting, standing, or walking at constant speeds on a motorized treadmill. Food and affection rewards were given for appropriate behaviors. Negative reinforcement was not used. The amplitude of stimulation was systematically varied up to a value supramaximal for Mwave amplitude (approximately twice the threshold for alpha motoneuron stimulation). The interval between consecutive stimuli was three seconds. None of the animals showed any sign of discomfort during these stimulation protocols. Analog signals from the various devices were recorded on magnetic tape (10 $\mathrm{kHz} /$ channel), digitally sampled at $20 \mathrm{kHz}$ (12 bit resolution) and stored in a microcomputer for later analysis. The evoked nerve CAP and muscle $M$-wave signals were sorted on the basis of stimulation intensity. Several trials were digitally averaged for each stimulus level and peak-to-peak amplitudes were computed.

\section{ResULTS}

\section{Ulnar nerve stimulation}

In the animal that had a bipolar stimulating cuff and a tripolar recording cuff on the ulnar nerve and indwelling EMG electrodes in the FCU muscle, a full range of stimulation amplitudes, from below threshold to supramaximal for $\mathrm{M}$-wave, was evaluated with the limb resting in a fixed position. The ulnar nerve CAP threshold was slightly lower than the FCU M-wave threshold, but once the M-wave was recruited the two waveforms grew proportionately. Examples of averaged sweeps recorded at progressively increasing stimulation levels are displayed in Figure 2. The triphasic shape $(+,-,+)$ of the ulnar nerve CAP waveform represents the summation of the temporally overlapping potentials contributed by all the individual nerve fibres recruited by the stimulus. Single nerve fibre action potentials are usually triphasic when recorded with tripolar nerve cuffs. ${ }^{7,11}$

With increasing stimulus intensity, the peak-to-peak amplitude of both the muscle M-wave and the nerve CAP demonstrated a sigmoidal dependence on the stimulus level. This is seen in Figures $3 \mathrm{~A}$ and $3 \mathrm{~B}$. Both the nerve CAP and muscle M-wave data sets were fit with the following four-parameter rational function:

$$
a+\frac{b(x-d)^{7}}{1+c(x-d)^{7}}
$$

The scatter about the curves was examined using the least squares method. Both the muscle $\mathrm{M}$-wave and nerve CAP data sets were fit very well by the sigmoidal curves ( $r$ values of .95 and .94 , respectively). Exponent values less than seven gave poorer fits. The close similarity in shape of these curves suggests that the nerve CAPs and M-waves had a similar dependence on stimulus intensity. A large fraction of the ulnar nerve

\section{FCU M-wave}
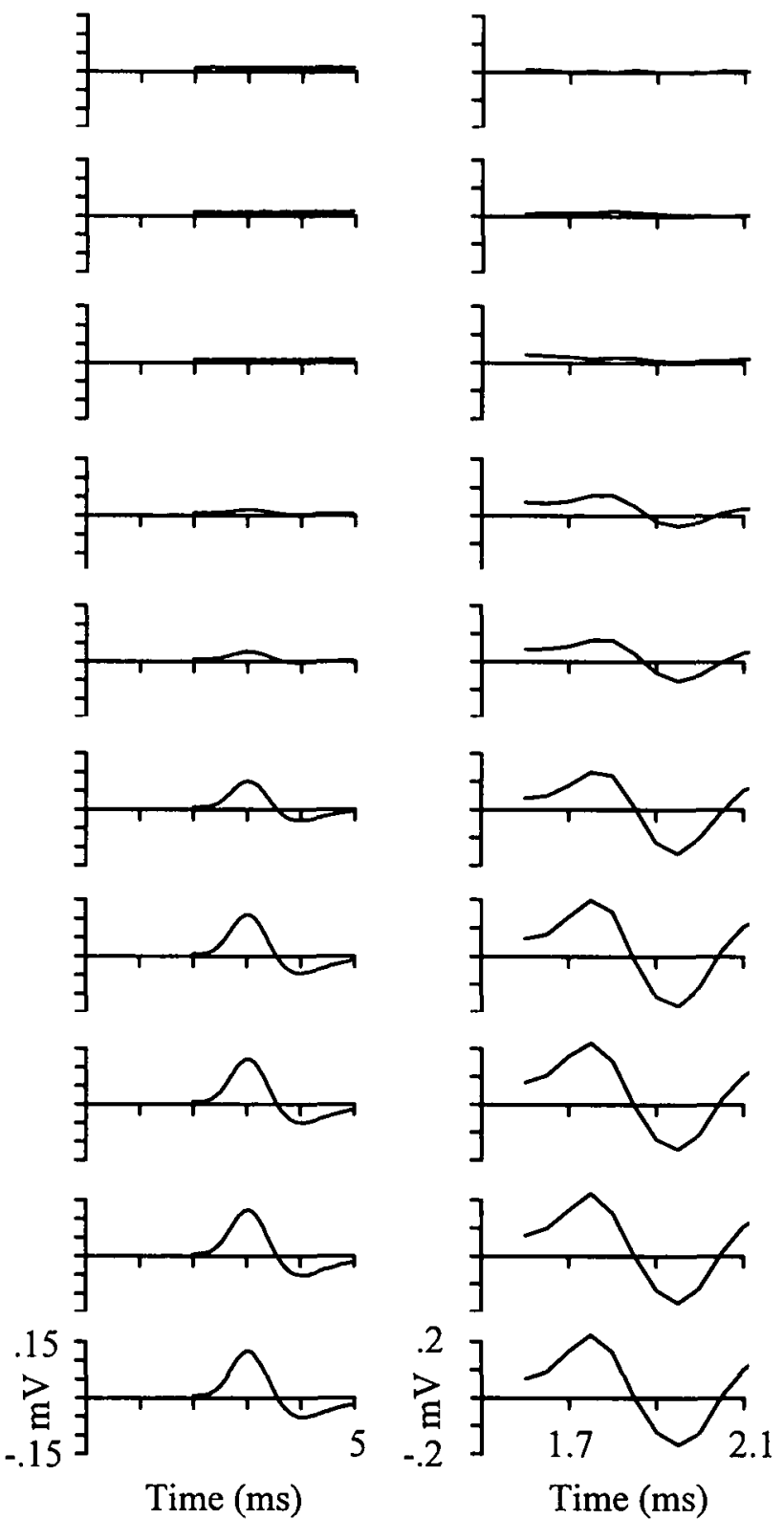

Figure 2: $M$-waves (left column) recorded in flexor carpi ulnaris (FCU) and nerve compound action potentials (right column) simultaneously recorded in the ulnar nerve during proximal ulnar nerve stimulation. The upper traces are at the lowest stimulation intensities; stimulus intensity increases as the traces proceed downward. All sweeps are averages ( $N$ between 18 and 141 ).

fibres was recruited over a narrow middle range of stimulus intensities. In this intermediate region, which is of particular interest in the present study, the relationship between the muscle $\mathrm{M}$-wave and the nerve CAP was well described by a straight line $(\mathrm{r}=.99, \mathrm{n}=53$; see Figure. $3 \mathrm{C})$.

\section{Tibial nerve stimulation}

Examples of sciatic nerve CAPs evoked with stimulation through the implanted tibial nerve cuff, and the corresponding 
$M$-waves recorded by the soleus muscle electrodes, are seen in Figure 4. As was seen for the ulnar nerve, the sciatic CAP waveforms (right column, Figure 4) are approximately triphasic $(+,-$, + ) and represent the summation of triphasic action potentials from single fibres. ${ }^{7.11}$ The latencies of the peaks of the triphasic sciatic CAP were approximately the same at the highest and lowest stimulation intensities (see Figure 4). This suggests that only group I axons were recruited in the tibial nerve throughout the range of stimulation intensities used.

The indwelling soleus EMG electrodes recorded complex waveforms during tibial nerve stimulation which included $M$ and $\mathrm{H}$-waves. In humans there is a large separation between the $\mathrm{M}$-wave and the $\mathrm{H}$-wave in the soleus EMG recorded during tibial nerve stimulation. However, because of the faster axonal conduction velocities and shorter distances in cat hindlimb nerves, there was no clear separation between the end of the $\mathrm{M}$-wave and the start of the $\mathrm{H}$-wave, which had onset latency between 6 and $8 \mathrm{~ms}$. The soleus M-wave amplitude measurements were done within a time window that excluded possible contributions by the early portion of the $\mathrm{H}$ wave.

In Figure 5, measurements from the same experiment as in Figure 4 are displayed. It can be seen that, except for the very early portion of the relationship, the correlation between soleus' $\mathrm{M}$-wave amplitude and sciatic CAP amplitude is well described by a straight line $(r=.98, n=93)$.

In one experiment, stimulation/recordings were made first during a period when the cat was standing quietly and repeated during an immediately subsequent period with the cat walking slowly on a treadmill. The $r$ value for the soleus $\mathrm{M}$-wave/sciatic CAP regression was $.94(n=151)$ during standing, and $.91(n=$ 216) during walking.

\section{Soleus muscle nerve stimulation}

Selective stimulation of the soleus muscle nerve through the implanted patch electrodes gave rise to a markedly different waveform in the sciatic nerve cuff recordings than that seen during tibial nerve stimulation. The right column in Figure 6 displays the recorded sciatic nerve cuff signals and the left column in Figure 6 shows corresponding EMG signals recorded with wire electrodes implanted in the belly of the soleus muscle. Two main negative going events, labeled $A$ and $B$, were recognized in the sciatic compound action potential. Event $A$ was recruited first and saturated at very low stimulation intensities, before there was a detectable M-wave. As stimulation intensity was further increased, event $B$ was progressively recruited in the sciatic compound action potential.

In the animal from which the data in Figure 6 were recorded, in postmortem measurements the soleus muscle patch and sciatic nerve cuff were separated by $9.5 \mathrm{~cm}$. This indicated that peak $\mathrm{A}$, which began $.86 \mathrm{~ms}$ poststimulus, contained fibres with conduction velocities up to $110 \mathrm{~m} / \mathrm{s}$. Peak B, which began $1.22 \mathrm{~ms}$ poststimulus, represented fibres with conduction velocities up to $79 \mathrm{~m} / \mathrm{s}$. Therefore, it was assumed that peak A represented the CAP produced by only large group I sensory afferent fibres, while peak $B$ included the recruitment of alpha-motoneuron fibres, which in the cat soleus are known to be of smaller diametre than group I sensory afferents. ${ }^{12}$

During soleus nerve stimulation, the amplitude of event $B$ was positively correlated with soleus $M$-wave amplitude $(r=0.92$,

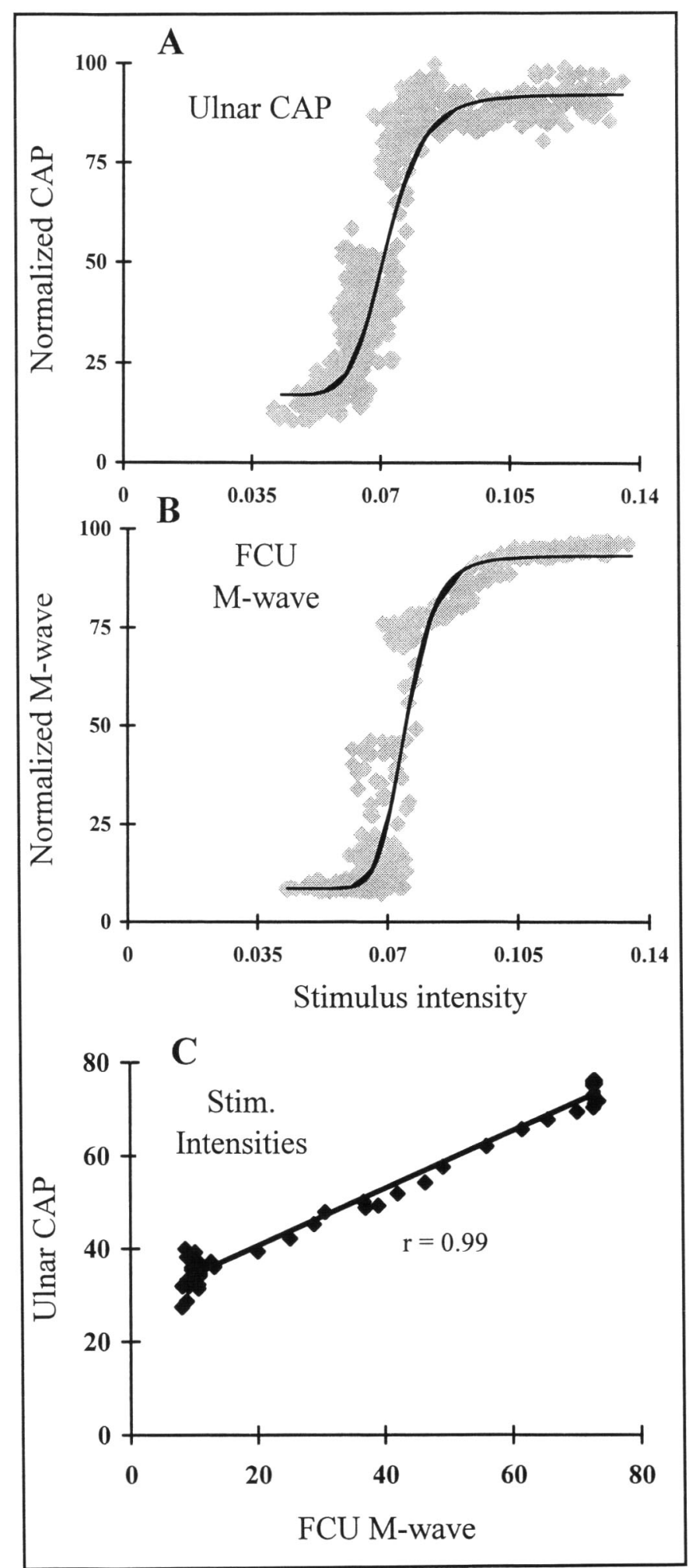

Figure 3. $A$ and $B$, peak-to-peak measurements of ulnar nerve CAPs and FCU $M$-waves, versus nerve cuff stimulus intensity, respectively. In each, the peak-to-peak measurements (values on the y-axis) have been normalized to the highest value attained. The curve shown in both is a four-parameter rational function (Eq. 1) fit to the data by the least squares method. C, Ulnar nerve CAPs plotted as a function of FCU Mwaves in the sensitive middle region of stimulation intensity. Units in both axes are normalized peak-to-peak measurements. 


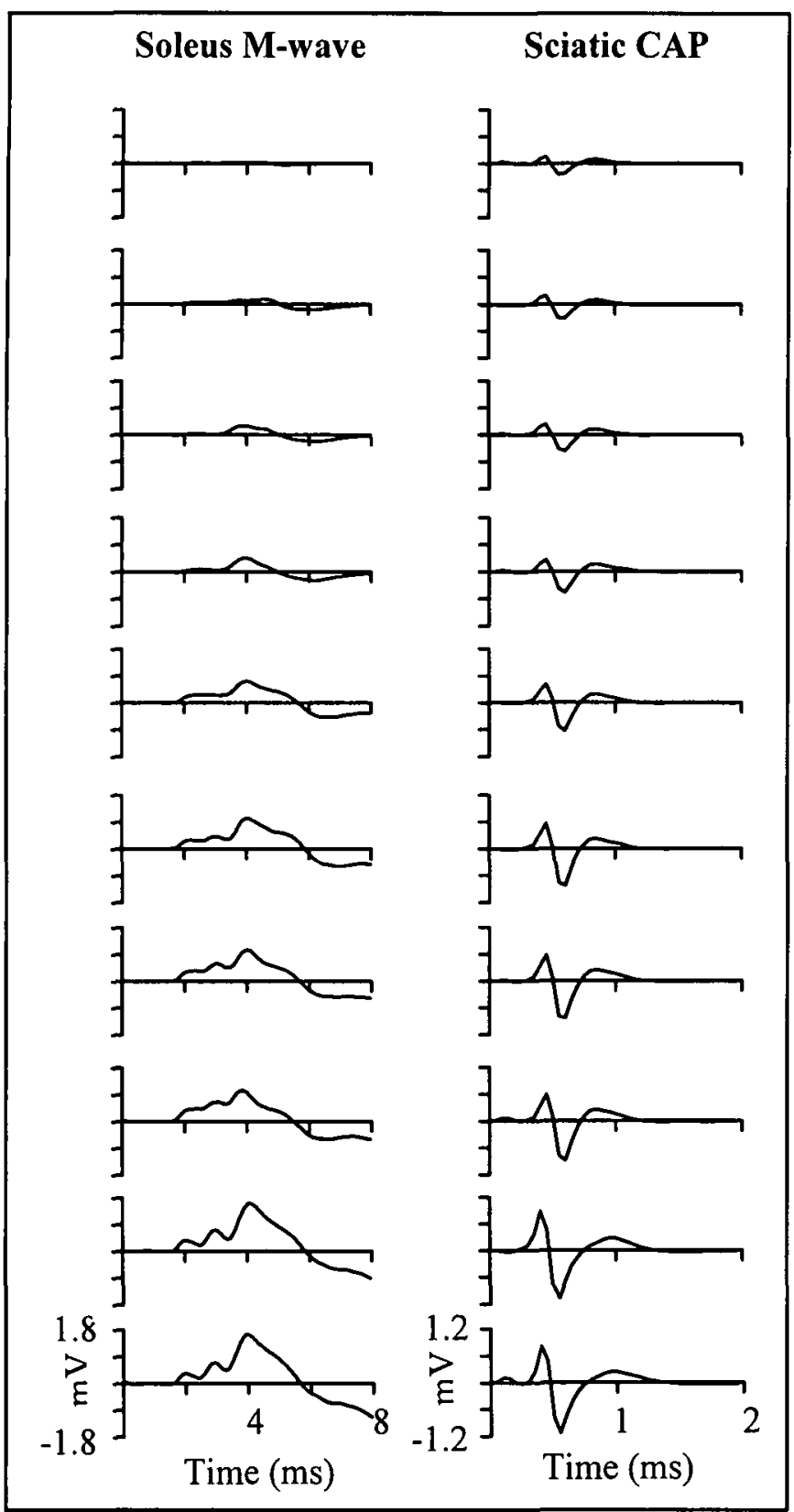

Figure 4: $M$-waves (left column) recorded in soleus muscle and nerve compound action potentials (right column) recorded in the sciatic nerve during tibial nerve stimulation. The upper traces are at the lowest stimulation intensities, and stimulus intensity increases as the traces proceed downward. All sweeps are averages ( $N$ between 3 and 46 ).

$\mathrm{n}=151$; see Figure 7B). Interestingly, the amplitude of event A was negatively correlated with soleus $M$-wave amplitude ( $r=$ $-0.62, n=151$; see Figure 7A). the latter result was considered to be artifactual and simply caused by a phase cancellation effect; i.e., the first positive phase of event $B$ overlapped with the main negative phase of event $A$, and partially cancelled it as event $B$ grew. If the recording cuff had been farther away from the stimulation site, this overlap would not have occurred. However, an additional possibility is that there may also have been a reduction in the number of recruited group I afferents at higher stimulation intensities due to formation of a virtual anode ${ }^{17}$ proximal

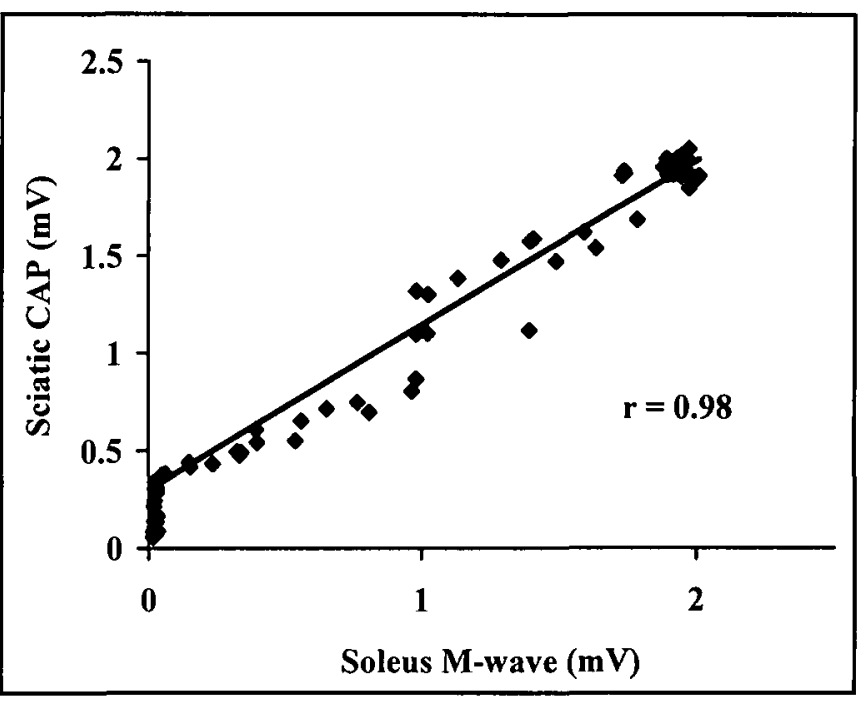

Figure 5: Peak-to-peak measurements of sciatic CAPs plotted as a function of soleus $M$-wave amplitude. Data were recorded during tibial nerve stimulation through a range of stimuli intensities from below $M$ wave threshold until above that required to attain a maximal $M$-wave.

to the cathode wire of the nerve cuff. In fact, adjustment of the blocking effect of the virtual anode has been used to generate unidirectionally propagating action potentials with a monopolar cuff. ${ }^{17}$

\section{Discussion}

This study has shown a generally very stable relationship between the centrally propagated nerve compound action potential (CAP) and the compound muscle potential (M-wave) evoked in a distal limb muscle by electrical stimuli. For the ulnar and tibial nerves, which have largely overlapping spectra of alpha motoneuron and group 1 sensory fibre diametres, ${ }^{12}$ the M-wave amplitude was closely correlated with the size of the centrally propagating CAP over a wide range of stimulation intensities. This can be taken to indicate that for these mixed nerves, the centripetal sensory nerve volley size is directly related to the efferent volley size. Although it is not possible to characterize the mixture of inputs recruited by increasing stimuli, this study has shown that the composite CAP volley is reproducible by controlling the $\mathrm{M}$-wave amplitude and it follows that, in particular, the number of group Ia afferents recruited (of prime interest in H-reflex studies) can also be controlled.

In addition, the small scatter found in the $\mathrm{M}$-wave/nerve CAP relationship for these nerves suggests that a fairly small number of matched $\mathbf{M}$-waves is sufficient to give a good estimate of the size of the centrally propagating afferent volley. Thus, for studies involving comparable nerves, the M-wave can provide an accurate measure of the stability of group I afferent fibre stimulation, a property that is particularly useful in human H-reflex experiments.

On the other hand, in experiments in which the cat soleus muscle nerve was stimulated, the $\mathrm{M}$-wave and the group I sensory nerve CAP were not well correlated. During soleus muscle nerve stimulation, two separate peaks were distinguished and could be independently measured in the sciatic nerve cuff 


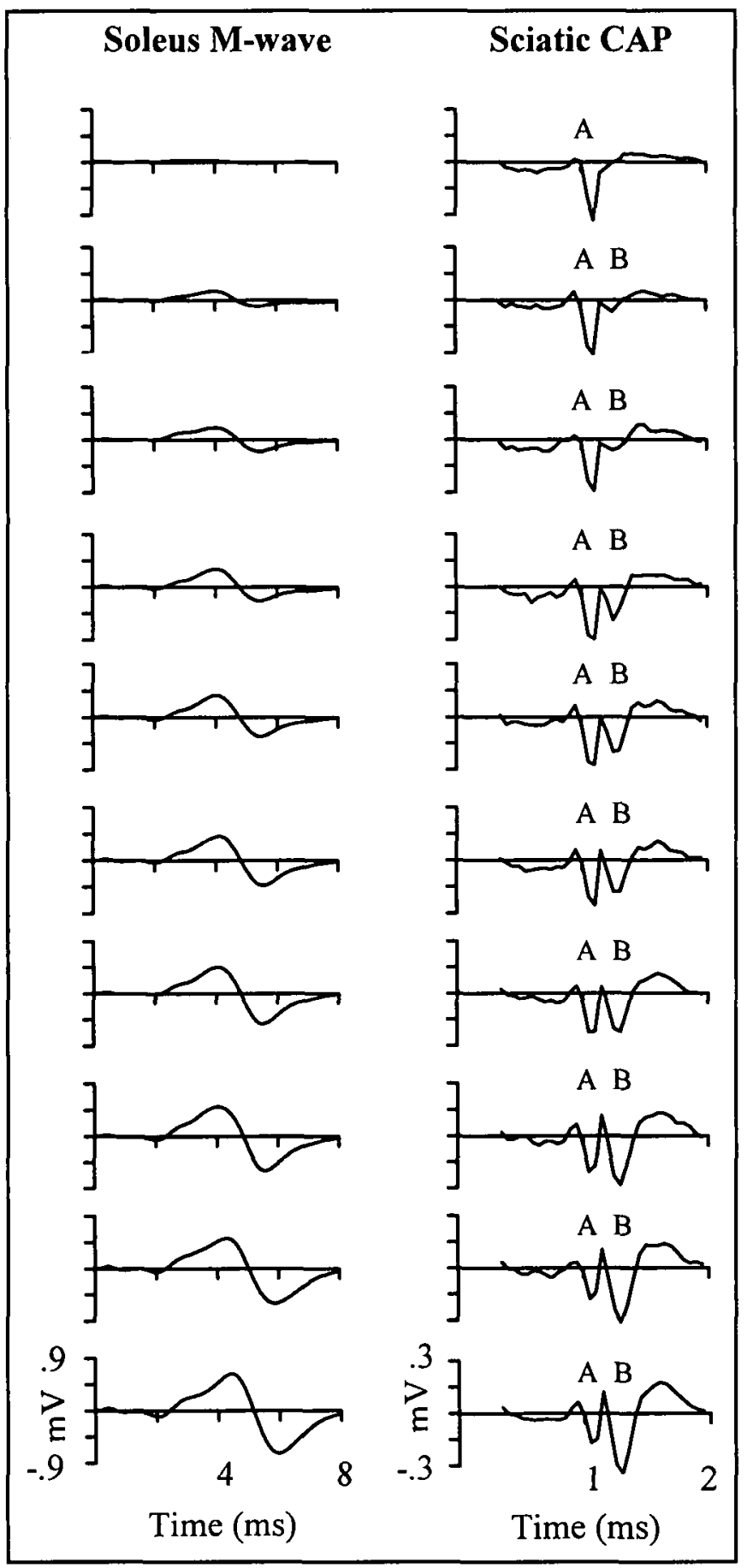

Figure 6: $M$-waves (left column) recorded in soleus muscle and nerve compound action potentials (right column) recorded in the sciatic nerve during soleus muscle nerve stimulation. The distinct negative peaks in the sciatic CAP are denoted as $A$ and $B$. The upper traces are at the lowest stimulation intensities, and stimulus intensity increases as the traces proceed downward. All sweeps are averages ( $N$ between 4 and 38 ).

record. The soleus muscle in the cat is composed of a nearly homogeneous population of slow muscle fibres ${ }^{15}$ innervated by type $\mathrm{S}$ motoneurons ${ }^{16}$ and the alpha-motoneuron fibres are distinctly smaller than the large diameter group la afferents. ${ }^{12}$ The present results (Figures 6 and 7) indicate that during soleus nerve stimulation, most of the group I sensory afferents were

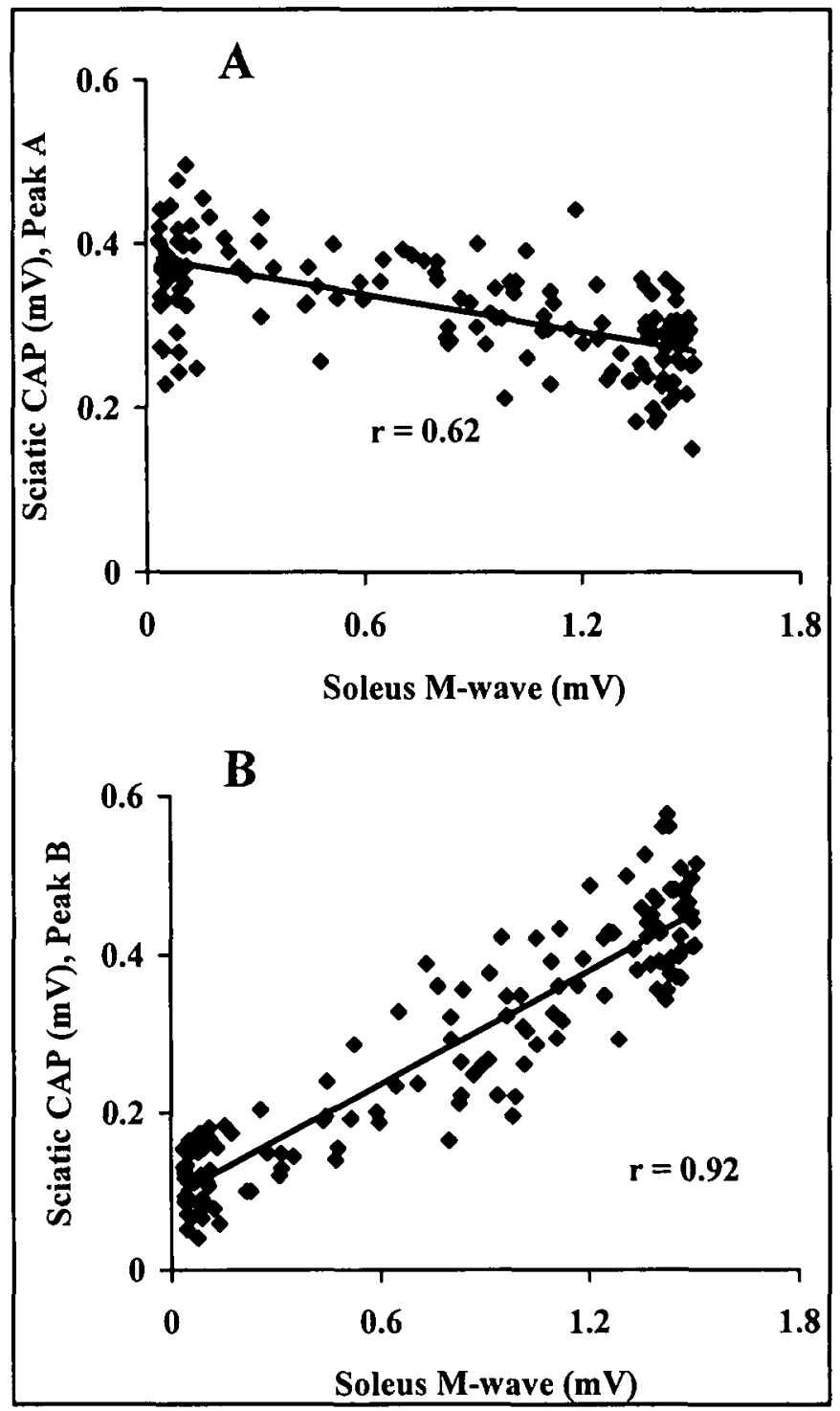

Figure 7: A, peak-to-peak measurements of the early event in the sciatic CAP (peak A in Figure 6) as a function of soleus $M$-wave amplitude during soleus nerve stimulation. $B$, peak-to-peak measurements of the later event in the sciatic CAP (peak $B$ in Figure 6) as a function of soleus $M$ wave amplitude during soleus nerve stimulation. The stimuli intensities ranged from subthreshold for $M$-wave to above that required for the maximal $M$-wave amplitude.

recruited before any $\mathrm{M}$-wave was recorded in the soleus muscle. It is concluded, therefore, that in studies involving this type of nerve fibre size distribution the $\mathrm{M}$-wave cannot be used to estimate the amplitude of the group I sensory volley evoked by electrical stimuli.

Given that the soleus nerve is a branch of the tibial nerve, the apparent contradiction in the results obtained with soleus vs. tibial nerve stimulation require further comment. Three points are relevant: 1) Compared to the soleus nerve, the tibial nerve contains wider ranges of group I sensory afferent axions (of Ia, Ib, cutaneous, and joint capsule origin) and alpha motor axons (innervating triceps surae, plantaris, other calf and foot muscles). The tibial nerve stimulation that resulted in roughly triphasic sciatic nerve CAPs reflected the graded recruitment of 
a continuum of group I axon sizes, with dominant contributions from the largest fibres. ${ }^{18}$ 2) The tibial nerve stimulating cuff location, approximately mid-way between the soleus nerve stimulating electrodes and the sciatic nerve recording cuff (see Figure 1), represented a shorter conduction distance to the sciatic cuff and this blurred the separation between afferent and motor fibre volleys that was so clearly seen when the soleus nerve was stimulated more distally (Figure 6). The possibility of resolving A and B events in Figure 4 was further eroded by the recruitment of many other axons of intermediate diametres with tibial nerve stimulation. 3) Finally, the soleus muscle $M$-wave provided a good prediction of afferent stimulation with tibial nerve stimulation, but not with soleus nerve stimulation, for the following reasons. When the tibial nerve was stimulated, the $\mathrm{M}$-wave recorded by the soleus muscle EMG electrodes no longer reflected exclusive recruitment of soleus motor units. It was contaminated with electrical activity evoked in motor units of other muscles, in particular the gastrocnemii and plantaris muscles which include large motor units with low thresholds to electrical stimulation of the tibial nerve. Cross-talk of potentials generated by motor units resident in one muscle can be clearly recorded by electrodes implanted in another muscle. ${ }^{19}$ In Figure 4, the first discernible change in $\mathrm{M}$-wave occurred less than $2 \mathrm{~ms}$ after tibial nerve stimulation, whereas in Figure 6 the onset occurred about 2 ms after soleus nerve stimulation even though the soleus stimulation point was much closer to the EMG recording electrodes (see Figure 1). Thus, the early $\mathrm{M}$-wave components in Figure 4 reflect the recuitment of motor units in muscles other than soleus. Crosstalk from motor units recruited in other muscles also produced larger M-wave amplitudes: the largest positive peak amplitude with tibial nerve stimulation was about $1.8 \mathrm{mV}$ (Figure 4) and less than $0.9 \mathrm{mV}$ with soleus nerve stimulation (Figure 6). Peakto-peak $\mathrm{M}$-wave values reached $2 \mathrm{mV}$ in Figure 5 , but did not reach $1.5 \mathrm{mV}$ in Figure 7.

On a separate note, this study showed that the relationship between the electrically evoked $M$-wave and the nerve CAP amplitude was largely independent of whether the animals sat quietly, stood or walked during stimulation. Moment to moment changes in muscle state are no doubt responsible for some variability in individual M-wave measurements obtained for closely matched sciatic CAP amplitudes. The EMG signal is strongly dependent on muscle geometry and changes in alignment of the electrodes relative to muscle fibres and in the shape of the muscle during movement may affect the EMG signal in complex ways. ${ }^{13}$ As well, M-waves may be potentiated during muscular contraction. ${ }^{14}$ In spite of these concerns, only modest differences in the variability of the soleus M-wave/sciatic CAP relationship were found for resting and walking conditions.

The relevance for human $\mathrm{H}$-reflex experiments of the present findings is straighforward. For typical mixed nerves (e.g., tibial or ulnar nerves) the $\mathrm{M}$-waves can provide a good measure of the centripetal stimulus stability. However, the present findings suggest that attempts at isolated stimulation of the human soleus muscle nerve, if successful, would preclude using the $\mathrm{M}$-wave as a measure of group Ia afferent recruitment efficacy. Therefore, although good theoretical reasons exist for attempting isolated stimulation of the human soleus muscle nerve during H-reflex testing, the standard approach of stimulating the triceps surae afferents in the tibial nerve higher in the popliteal fossa, remains a better option and the resulting triceps surae $\mathbf{M}$-wave can be used as a valuable measure of stimulus efficacy.

\section{ACKNOWLEDGEMENTS}

We thank M. Maitland and T. Razniewska for assistance with training and surgical preparation of cats at The University of Calgary, where most of the data for this study were collected, and K. Strange for providing access for data collection in an implanted animal at Simon Fraser University. This work was supported by grants from MRC and Rick Hansen "Man In Motion" Legacy Fund to JAH and a Postdoctoral Fellowship from Alberta Paraplegic Foundation to GIB.

\section{REFERENCES}

1. Garrett M, Ireland A, Luckwill RG. Changes in excitability of the Hoffman reflex during walking in man. J Physiol 1984; 355: 23p.

2. Capaday C, Stein RB. Amplitude modulation of the soleus H-reflex in the human during walking and standing. J Neurosci 1986; 6: 1308-1313.

3. Crenna P, Frigo C. Excitability of the soleus H-reflex arc during walking and stepping in man. Exp Brain Res 1987; 66: 49-60.

4. Wolpaw JR. Operant conditioning of primate spinal reflexes: the $\mathrm{H}$ reflex. J Neurophysiol 1987; 57: 443-459.

5. Dietz V, Faist F, Pierrot-Deseilligny E. Amplitude modulation of the quadriceps $\mathrm{H}$-reflex in the human during the early stance phase of gait. Exp Brain Res 1990; 79: 221-224.

6. Boorman G, Becker W, Morrice B, Lee RG. Modulation of the soleus $\mathrm{H}$-reflex during pedalling in normal subjects and in patients with spinal cord injury. J Neurol Neurosurg Psychiatry 1992; 55: 1150-1156.

7. Hoffer JA. Techniques to record spinal cord, peripheral nerve and muscle activity in freely moving animals. $n$ : AA Boulton, GB Baker and $\mathrm{CH}$ Vanderwold, eds. Neurophysiological Techniques: Applications to Neural Systems. Neuromethods, Vol 15, Clifton NJ, Humana Press 1990: 65-145.

8. McNeal DR, Baker L, Symons JT. Recruitment data for nerve cuff electrodes: implications for design of implantable stimulators. IEEE Trans Biomed Eng 1989; 36: 301-308.

9. Fang Z, Mortimer JT. Selective activation of small motor axons by quasitrapezoidal current pulses. IEEE Trans Biomed Eng 1991; 38: $168-174$

10. Mortimer JT. Motor Prostheses. In: VB Brooks, ed. Handbook of Physiology, The Nervous System II: Motor Control, Bethesda, Maryland, Am Physiol Soc 1981; 155-187.

11. Marks WB, Loeb GE. Action currents, internodal potentials and extracellular records of myelinated mammalian nerve fibres derived from node potentials. Biophys J 1976; 16: 655-668.

12. Boyd IA, Davey MR. Composition of Peripheral Nerves. Edinburgh and London, E \& S Livingstone Ltd. 1968: 41.

13. Loeb GE, Gans J. Electromyography for Experimentalists. Chicago, University of Chicago Press 1986.

14. Hicks A, Fenton J, Garner S, McComas AJ. M-wave potentiation during and after muscle activity. J Appl Physiol 1989; 66: 2606-2610.

15. Ariano MA, Armstrong RB, Edgerton VR. Hindlimb muscle fiber populations of five mammals. J Histochem Cytochem 1973; 21: 51-55.

16. Burke RE. Motor units: anatomy, physiology and functional organization. In: Brooks VB, ed. Handbook of Physiology, Sect I. The Nervous System, Vol 2. Motor Control. Bethesda MD, American Physiological Society 1981: 345-423.

17. Ungar IJ, Mortimer JT, Sweeney JD. Generation of unidirectionally propagating action potentials using a monopolar electrode cuff. Annals Biomed Eng 1986; 14: 437-450.

18. Hoffer JA, Loeb GE, Pratt CA. Single unit conduction velocities from averaged nerve cuff electrode records in freely moving cats. J Neurosci Methods 1981; 4: 211-225.

19. Hoffer JA, Loeb GE, Marks WB, et al. Cat hindlimb mononeurons during locomotion. I. Destination, axonal conduction velocity and recruitment threshold. J Neurophysiol 1987; 57: 530-553. 